\title{
Inverted velocity profiles in rarefied cylindrical Couette gas flow and the impact of the accommodation coefficient
}

\author{
Sun Yuhong, Robert W. Barber, and David R. Emerson ${ }^{\text {a) }}$ \\ Centre for Microfluidics and Microsystems Modelling, CCLRC Daresbury Laboratory, \\ Warrington WA4 4AD, United Kingdom
}

(Received 14 June 2004; accepted 18 January 2005; published online 10 March 2005)

\begin{abstract}
Cylindrical Couette gas flow in the noncontinuum regime has been investigated using the boundary treatment derived from Maxwell's slip-flow model. It is shown that the tangential momentum accommodation coefficient plays an important role in determining the predicted velocity profile. The present analysis is in close agreement with previous analytical studies and shows good qualitative agreement with available direct simulation Monte Carlo data. The results predict the presence of an inverted velocity profile for the case where the inner cylinder rotates and the outer cylinder is stationary. However, our analysis further shows that the phenomenon of velocity inversion is only dependent on the accommodation coefficient of the outer cylinder. From the analysis, we derive specific criteria for the accommodation coefficient of the outer cylinder under which (i) no velocity inversion will take place, (ii) a partially inverted velocity profile will be observed, and (iii) a fully inverted velocity profile will be present. In contrast, when the outer cylinder rotates and the inner cylinder is stationary, it is shown that velocity inversion does not occur. () 2005 American Institute of Physics. [DOI: 10.1063/1.1868034]
\end{abstract}

\section{INTRODUCTION}

Couette flow between two concentric rotating cylinders is a classical fluid dynamics problem discussed in many textbooks. However, recent analytical and numerical studies have demonstrated that under certain conditions of rarefaction, the flow between concentric cylinders can exhibit completely nonintuitive behavior. For example, in the case of a stationary outer cylinder and a rotating inner cylinder, the velocity profile can become "inverted" with the velocity increasing from the inner rotating wall to the outer stationary wall. The phenomenon of velocity inversion was first predicted by Einzel, Panzer, and Liu (EPL) ${ }^{1}$ who developed a generalized slip-boundary condition for incompressible flow over curved or rough surfaces. EPL did not specifically consider a rarefied gas, and instead, analyzed the flow by invoking the concept of a slip length. Applying their generalized slip-boundary condition to cylindrical Couette flow, EPL predicted that the velocity profile would become inverted for large values of slip length.

Tibbs $e t a l .^{2}$ subsequently recast the EPL formulation so that it was directly applicable to a rarefied gas. This was achieved by defining the slip length, $\zeta_{0}=\alpha(2 / \sigma-1) \lambda$ where $\lambda$ is the mean-free path of the gas molecules, $\alpha \approx 1.15$ and $\sigma$ is the tangential momentum accommodation coefficient (TMAC) which can vary from zero (for specular reflection) up to unity (for complete or diffuse accommodation). Tibbs et al. considered argon under STP conditions $(\lambda=6.25$ $\times 10^{-8} \mathrm{~m}$ ) for a stationary outer cylinder and an inner cylinder rotating at an angular velocity $\omega$ of $5.17 \times 10^{8} \mathrm{rad} / \mathrm{s}$ (cor-

\footnotetext{
${ }^{\text {a) }}$ Author to whom correspondence should be addressed. Telephone: +44 (0)1925 603221. Fax: +44 (0)1925 603634. Electronic mail: d.r.emerson@dl.ac.uk
}

responding to a Mach number of 0.3). They presented velocity profiles for a range of accommodation coefficients and found that the velocity inversion process only occurred at small values of TMAC. The results from their direct simulation Monte Carlo (DSMC) approach showed that the velocity profiles predicted by the EPL formulation were generally in good agreement with their DSMC data. Moreover, the DSMC simulations showed that there was less than a $1 \%$ variation in density throughout the flow domain, suggesting that compressibility effects may not be important for this particular problem.

Cylindrical Couette flow has also been studied by Aoki et $a l^{3}$ using several alternative approaches: a systematic asymptotic analytical solution at small Knudsen numbers, a direct simulation Monte Carlo method, and a direct numerical solution of the Boltzmann equation using a finite difference method based on the Bhatnagar-Gross-Krook (BGK) approximation. The results again confirmed the existence of an inverted velocity profile for small values of accommodation coefficient and also showed that the occurrence of velocity inversion could be related to a critical accommodation coefficient. It is well known that the near-continuum Maxwell slip velocity becomes infinitely large when the accommodation coefficient approaches zero. However, for Couette flow, Aoki et al. developed a rigorous asymptotic analysis of the Boltzmann equation to obtain the flow properties at small Knudsen number $(\mathrm{Kn})$ and small accommodation coefficient (of the order of $\mathrm{Kn}$ ). They proved that Maxwell's diffusespecular treatment was valid for small $\sigma$ and that the fluiddynamic system obtained was equivalent to the compressible Navier-Stokes equations. Moreover, Aoki et al. showed that if the rotation speed was small (but much greater than $\mathrm{Kn}$ ), the system obtained was equivalent to the incompressible Navier-Stokes equations and could be used to derive an ana- 
lytical solution. The compressible solution obtained by Aoki et $a l^{3}{ }^{3}$ was in good agreement with their DSMC data up to $\mathrm{Kn}=0.1$. Furthermore, they found no appreciable difference between their compressible and incompressible solutions up to a Mach number of 0.5 , adding further support to the findings of Tibbs et al. ${ }^{2}$

The present investigation provides an independent reassessment of the velocity inversion process in cylindrical Couette flows. Instead of implementing the EPL slip-length approach, the current analysis uses Maxwell's ${ }^{4}$ slip-boundary condition to define the velocity discontinuity between the gas and the solid walls. Two fundamental cylindrical Couette flow cases are considered: a stationary outer cylinder and a rotating inner cylinder (case I); and, second, a stationary inner cylinder and a rotating outer cylinder (case II). Previous analytical studies ${ }^{1,2,5,6}$ have generally assumed that the accommodation coefficients at the inner and outer cylinder walls are identical. In contrast, the present study considers the influence of both the inner and outer accommodation coefficients. The paper then defines limiting analytical criteria that can be used to predict whether an inverted, partially inverted or normal velocity profile will occur.

\section{PROBLEM FORMULATION}

The present investigation considers isothermal, rarefied Couette flow between two concentric rotating cylinders. The inner and outer cylinders have radii $R_{1}$ and $R_{2}$ and rotate at angular velocities $\omega_{1}$ and $\omega_{2}$, respectively. In a cylindricalpolar coordinate $(r, \theta)$ reference frame, the circumferential momentum expression of the incompressible Navier-Stokes equations can be written for rotating Couette flow as (Schlichting ${ }^{7}$ )

$$
\frac{d^{2} u_{\theta}}{d r^{2}}+\frac{d}{d r}\left(\frac{u_{\theta}}{r}\right)=0
$$

where $u_{\theta}$ is the tangential velocity component and $r$ is the radius. For low Knudsen numbers and moderate Mach numbers, the assumption that the flow is incompressible is reasonable. ${ }^{2,3}$ Moreover, at $\mathrm{Kn}=0.5$, the DSMC results of Tibbs et $a l^{2}$ showed that temperature variations for this problem were less than $3 \%$ over the entire domain, further suggesting that the isothermal assumption is also reasonable.

Under rarefied conditions, a slip-velocity boundary condition has to be applied at the inner and outer rotating walls. In the absence of thermal slip, Maxwell's viscous slipvelocity boundary condition can be written ${ }^{8,9}$ as

$$
u_{\text {slip }}=u_{\text {gas }}-u_{\text {wall }}= \pm \alpha \frac{(2-\sigma)}{\sigma} \frac{\lambda}{\mu} \tau_{\text {wall }},
$$

where $\mu$ is the viscosity and $\tau$ is the shear stress. If the mean-free path is defined as $\lambda=(\mu / P) \sqrt{\pi R T / 2}$, as in Maxwell's original analysis, the value of $\alpha$ is unity. However, more rigorous kinetic analyses of the Boltzmann equation for planar flows ${ }^{10-12}$ have subsequently shown that the value of $\alpha$ for fully diffusive reflection, based on Maxwell's definition of the mean-free path, is very close to $1.016 \times 2 / \sqrt{\pi} \approx 1.15$. The "exact" leading coefficient of 1.016191 was obtained numerically by Loyalka et al. ${ }^{10}$ using a BGK model of the
Boltzmann equation whereas Wakabayashi et al. ${ }^{11}$ solved the linearized Boltzmann equation and obtained a value of 0.98737. In practice, $\alpha$ has been shown to depend upon the accommodation coefficient ${ }^{10-12}$ but for ease of analysis in the present study, $\alpha$ is assumed to be unity in common with many experimental and numerical hydrodynamic approaches. $^{13-15}$

For rotating Couette flow, Eq. (2) can be written in cylindrical-polar form as

$$
u_{\theta(g a s)}-u_{\theta(\text { wall })}= \pm\left.\frac{(2-\sigma)}{\sigma} \lambda\left(\frac{d u_{\theta}}{d r}-\frac{u_{\theta}}{r}\right)\right|_{\text {wall }} .
$$

The term $u_{\theta} / r$ on the right hand side of Eq. (3) arises from the fact that the slip velocity is directly proportional to the wall shear stress. Unfortunately, this term is often (although not always ${ }^{3,8,9}$ ) neglected when analyzing slip flows on nonplanar surfaces. Application of Eq. (3) to the inner and outer cylinder walls leads to

$$
\left.u_{\theta}\right|_{r=R_{1}}=\omega_{1} R_{1}+\left.\frac{\left(2-\sigma_{1}\right)}{\sigma_{1}} \lambda\left(\frac{d u_{\theta}}{d r}-\frac{u_{\theta}}{r}\right)\right|_{r=R_{1}}
$$

and

$$
\left.u_{\theta}\right|_{r=R_{2}}=\omega_{2} R_{2}-\left.\frac{\left(2-\sigma_{2}\right)}{\sigma_{2}} \lambda\left(\frac{d u_{\theta}}{d r}-\frac{u_{\theta}}{r}\right)\right|_{r=R_{2}} .
$$

Following the notation adopted by Einzel et al., ${ }^{1}$ the general solution for the velocity profile can be written as

$$
u_{\theta}(r)=a r+\frac{b}{r}
$$

where

$$
a=\frac{A \omega_{1}-B \omega_{2}}{A-B}, \quad b=\frac{\omega_{1}-\omega_{2}}{B-A} .
$$

The parameters $A$ and $B$ can be derived as

$$
A=\frac{1}{R_{2}^{2}}\left(1-\frac{\left(2-\sigma_{2}\right)}{\sigma_{2}} \frac{2 \lambda}{R_{2}}\right), \quad B=\frac{1}{R_{1}^{2}}\left(1+\frac{\left(2-\sigma_{1}\right)}{\sigma_{1}} \frac{2 \lambda}{R_{1}}\right) .
$$

As previously discussed, two cylindrical Couette flow problems are considered in the present study. For case I, with a stationary outer cylinder $\left(\omega_{2}=0\right)$ and a rotating inner cylinder, Eq. (6) can be written in nondimensional form as

$$
\begin{aligned}
u_{\theta}^{*}=\frac{u_{\theta}}{\omega_{1} R_{1}} & =\frac{A}{(A-B)} \frac{r}{R_{1}}+\frac{1}{(B-A) R_{1} r} \\
& =\frac{1}{(A-B) R_{1}}\left(A r-\frac{1}{r}\right),
\end{aligned}
$$

whereas for case II, with a stationary inner cylinder $\left(\omega_{1}\right.$ $=0$ ) and a rotating outer cylinder, Eq. (6) becomes 


$$
\begin{aligned}
u_{\theta}^{*}=\frac{u_{\theta}}{\omega_{2} R_{2}} & =\frac{B}{(B-A)} \frac{r}{R_{2}}+\frac{1}{(A-B) R_{2} r} \\
& =\frac{1}{(B-A) R_{2}}\left(B r-\frac{1}{r}\right) .
\end{aligned}
$$

In the case of continuum flow, the coefficients $A$ and $B$ in Eq. (8) simplify to $A=1 / R_{2}^{2}$ and $B=1 / R_{1}^{2}$. The velocity profile for continuum flow can then be expressed in terms of the ratio of the cylinder radii, $\chi=R_{1} / R_{2}$ :

case I, $\quad u_{\theta}(r)=\frac{\chi^{2}}{\left(\chi^{2}-1\right)} \omega_{1} r+\frac{R_{1}^{2}}{\left(1-\chi^{2}\right)} \frac{\omega_{1}}{r}$,

case II, $\quad u_{\theta}(r)=\frac{1}{\left(1-\chi^{2}\right)} \omega_{2} r+\frac{\chi^{2} R_{2}^{2}}{\left(\chi^{2}-1\right)} \frac{\omega_{2}}{r}$.

Although Eqs. (11) and (12) are similar in form, it is well known that for continuum cylindrical Couette flow, the velocity profile varies strongly with the ratio $R_{1} / R_{2}$ for case I while the velocity profile is almost independent of the ratio for case II (Schlichting ${ }^{7}$ ).

\section{THE PHENOMENON OF VELOCITY INVERSION}

To illustrate the phenomenon of velocity inversion, we consider case I (inner cylinder rotating). Following Tibbs et $a l .{ }^{2}$ the radii of the inner and outer cylinders are chosen to be $3 \lambda$ and $5 \lambda$, respectively, and the accommodation coefficients are assumed to be equal at the inner and outer cylinders. Figure 1(a) presents a comparison between the analytical solution and the DSMC data obtained by Tibbs et al. ${ }^{2}$ Exact quantitative agreement between the DSMC results and the analytical model is not expected, since the separation distance between the cylinder walls is only two molecular mean-free paths, implying a Knudsen number, $\mathrm{Kn}=\lambda /\left(R_{2}\right.$ $-R_{1}$ ) of 0.5 . At this Knudsen number, the Navier-Stokes equations are beyond the limit of their theoretical applicability. Nevertheless, the results show that the DSMC data and the analytical formulation follow the same basic trends and predict an inverted velocity profile for an accommodation coefficient of 0.1 .

In contrast, Fig. 1(b) presents a comparison between the DSMC data and an incorrect boundary treatment where the $u_{\theta} / r$ term in Eq. (3) has been neglected. Failure to account for this component leads to important differences in the predicted velocity profiles, with essential information being lost. It can be seen that the incorrect analytical solution is unable to predict the velocity inversion phenomenon, clearly highlighting the importance of including the $u_{\theta} / r$ term.

It is interesting to note that, for the specific case of the accommodation coefficients of the inner and outer cylinders having the same value, the family of velocity profiles in Fig. 1(a) all pass through a common point that is independent of the value of the accommodation coefficient. The location of the point can be derived analytically using Eq. (9) and is given by

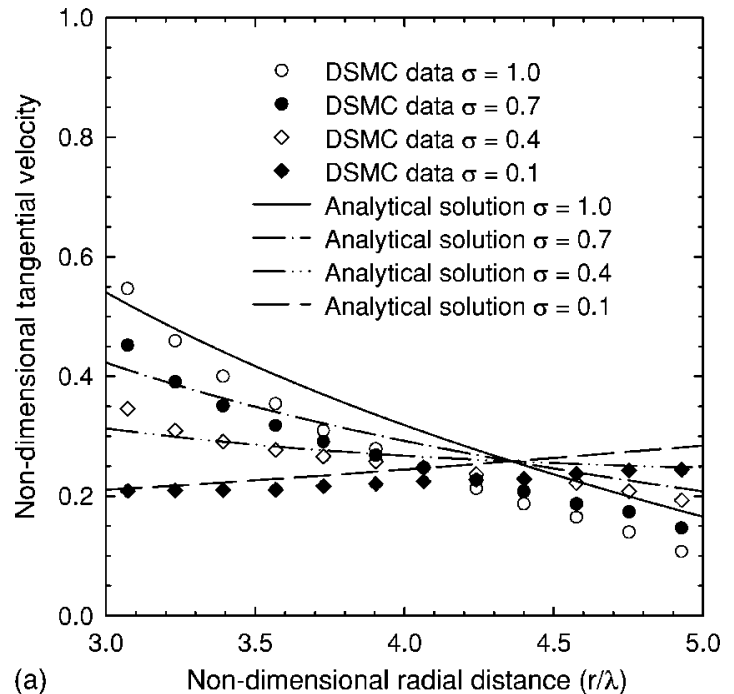

(a) Non-dimensional radial distance $(r / \lambda)$

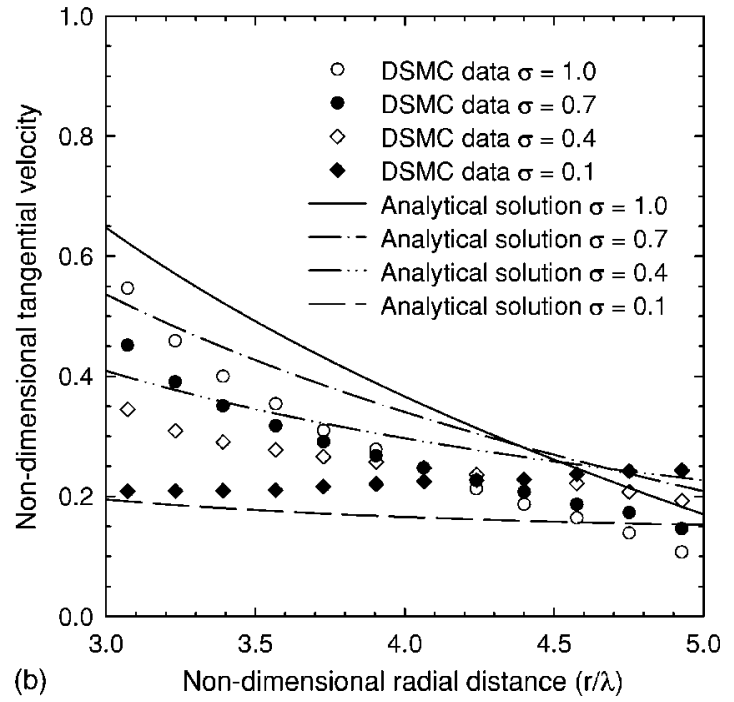

FIG. 1. Nondimensional velocity vs radial distance for $\sigma_{1}=\sigma_{2}=\sigma$. (a) Correct solution using the shear stress boundary treatment defined in Eq. (3). (b) Incorrect solution obtained by neglecting the $u_{\theta} / r$ term in Eq. (3).

$$
r=\sqrt{\frac{R_{1}^{3}+R_{2}^{3}}{R_{1}+R_{2}}} .
$$

For the problem under consideration, the location is given by $r / \lambda=\sqrt{19}$ and the nondimensional velocity has the value 0.258. It is evident that the DSMC data also show the same phenomenon although the location and value is slightly different.

\section{VELOCITY INVERSION CRITERIA}

In the following sections, we identify the limiting analytical criteria that will predict whether a normal or an inverted velocity profile will occur. We will also introduce the concept of a "partially inverted" velocity profile that, for case I, means the velocity will initially decrease away from the inner cylinder and then increase towards the outer cylinder. The impact of the TMAC at the inner and outer walls will also be considered. 


\section{A. Investigation of case I}

Equation (9) shows that the velocity is inversely proportional to $(A-B)$, with the definition of $A$ and $B$ given by Eq. (8). The velocity gradient can now be used as a criterion to judge whether the function is increasing or decreasing with radial distance $r$. For clarity, the superscript $*$ denoting a nondimensional velocity is omitted from the subsequent analysis. The gradient is therefore given by

$$
\frac{d u_{\theta}}{d r}=\frac{1}{(A-B) R_{1}}\left(A+\frac{1}{r^{2}}\right) .
$$

From Eq. (8), we can obtain the following expression for $(A-B)$ :

$$
\begin{aligned}
A-B= & -\frac{1}{R_{1}^{2} R_{2}^{2}}\left[\left(R_{2}^{2}-R_{1}^{2}\right)+R_{1}^{2} \frac{\left(2-\sigma_{2}\right)}{\sigma_{2}} \frac{2 \lambda}{R_{2}}\right. \\
& \left.+R_{2}^{2} \frac{\left(2-\sigma_{1}\right)}{\sigma_{1}} \frac{2 \lambda}{R_{1}}\right] .
\end{aligned}
$$

Since $R_{1}<R_{2}$, the term $(A-B)$ is always negative. For case I, a noninverted velocity profile means that the velocity will decrease monotonically from the inner to the outer cylinder. This implies $d u_{\theta} / d r<0$ for the whole range, $R_{1} \leqslant r \leqslant R_{2}$. Conversely, if $d u_{\theta} / d r>0$ throughout the range $R_{1} \leqslant r \leqslant R_{2}$, a fully inverted velocity profile will exist. From Eq. (14), this requirement can only be satisfied when $A+r^{-2}<0$. We know that $A+R_{2}^{-2} \leqslant A+r^{-2} \leqslant A+R_{1}^{-2}$, and therefore if $A+R_{1}^{-2}<0$ is true, then $A+r^{-2}<0$ will always be satisfied. This requirement yields

$$
A+\frac{1}{R_{1}^{2}}=\frac{1}{R_{1}^{2} R_{2}^{2}}\left[R_{1}^{2}+R_{2}^{2}-\frac{\left(2-\sigma_{2}\right)}{\sigma_{2}} \frac{2 \lambda R_{1}^{2}}{R_{2}}\right]<0,
$$

which holds if

$$
\sigma_{2}<2\left(1+\frac{\left(R_{1}^{2}+R_{2}^{2}\right) R_{2}}{2 \lambda R_{1}^{2}}\right)^{-1} .
$$

If Eq. (17) is satisfied, a fully inverted velocity profile will always be observed and this phenomenon is independent of the value of the accommodation coefficient at the inner cylinder. For the specific case when the inner and outer cylinder radii are $3 \lambda$ and $5 \lambda$, respectively, Eq. (17) provides an upper bound for a fully inverted velocity profile, given by $\sigma_{2}<9 / 47 \approx 0.1915$. This is illustrated in Fig. 2 , which also shows that the accommodation coefficients at the inner and outer cylinders have an opposite effect on the magnitude of the inverted velocity, i.e., as $\sigma_{2}$ is increased $u_{\theta}$ decreases, but as $\sigma_{1}$ is increased $u_{\theta}$ increases.

If the condition $d u_{\theta} / d r<0$ holds throughout $R_{1} \leqslant r$ $\leqslant R_{2}$, the velocity will be a decreasing function of $r$ and no inverted velocity profile is observed. This is obtained when $A+r^{-2}>0$. Again, using the fact that $A+R_{2}^{-2} \leqslant A+r^{-2} \leqslant A$ $+R_{1}^{-2}$, then if $A+R_{2}^{-2}>0$ is valid, $A+r^{-2}>0$ will always be satisfied. We therefore need to satisfy the following expression for no inverted velocity profile to occur:
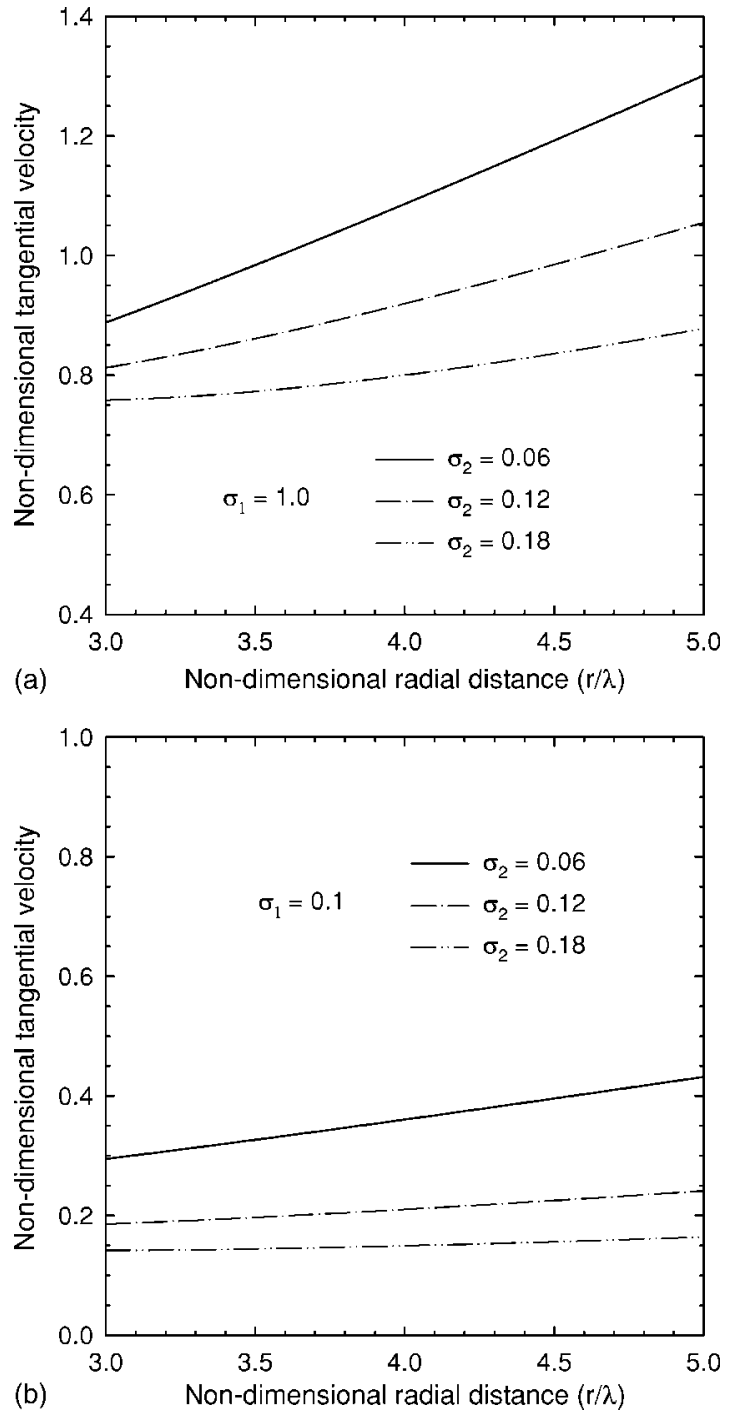

FIG. 2. Nondimensional velocity profiles when the accommodation coefficient at the stationary outer cylinder is less than $9 / 47$.

$$
A+\frac{1}{R_{2}^{2}}=\frac{1}{R_{2}^{2}}\left[2-\frac{\left(2-\sigma_{2}\right)}{\sigma_{2}} \frac{2 \lambda}{R_{2}}\right]>0 .
$$

The condition for Eq. (18) to hold is

$$
\sigma_{2}>2\left(1+\frac{R_{2}}{\lambda}\right)^{-1} .
$$

For the current case, the limiting value given by Eq. (19) is $\sigma_{2}>1 / 3$. Figure 3 shows that if the accommodation coefficient at the outer wall is greater than $1 / 3$, the velocity profile always decreases monotonically, even for very small values of the inner wall accommodation coefficient. It is also observed that increasing the accommodation coefficient at the inner wall causes the velocity to increase.

The final condition that needs to be considered is the case $d u_{\theta} / d r=0$. When this situation occurs, it implies 

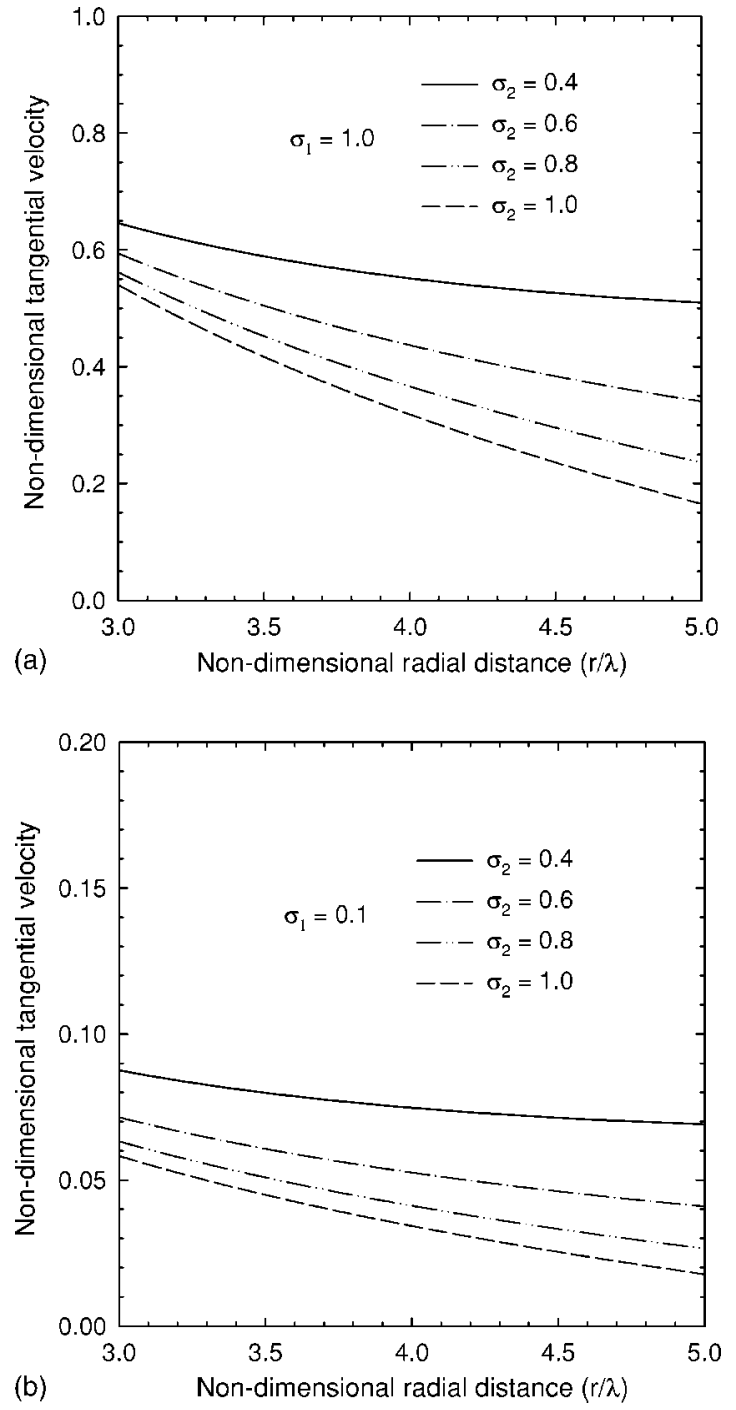

FIG. 3. Nondimensional velocity profiles when the accommodation coefficient at the stationary outer cylinder is greater than $1 / 3$.

$$
A+\frac{1}{r^{2}}=0
$$

Substituting for $A$ from Eq. (8) into Eq. (20) gives the following solution:

$$
r^{*}=\sqrt{\frac{R_{2}^{2}}{\frac{\left(2-\sigma_{2}\right)}{\sigma_{2}} \frac{2 \lambda}{R_{2}}-1}} .
$$

For Eq. (21) to have a real root, the denominator must be greater than zero. However, to ensure $r^{*}<R_{2}$ the denominator must be greater than unity. We therefore require that

$$
\sigma_{2}<2\left(1+\frac{R_{2}}{\lambda}\right)^{-1}
$$

The second derivative of velocity at the point $r^{*}$ indicates whether the velocity has a maximum or minimum value. Using Eq. (14), the second derivative is
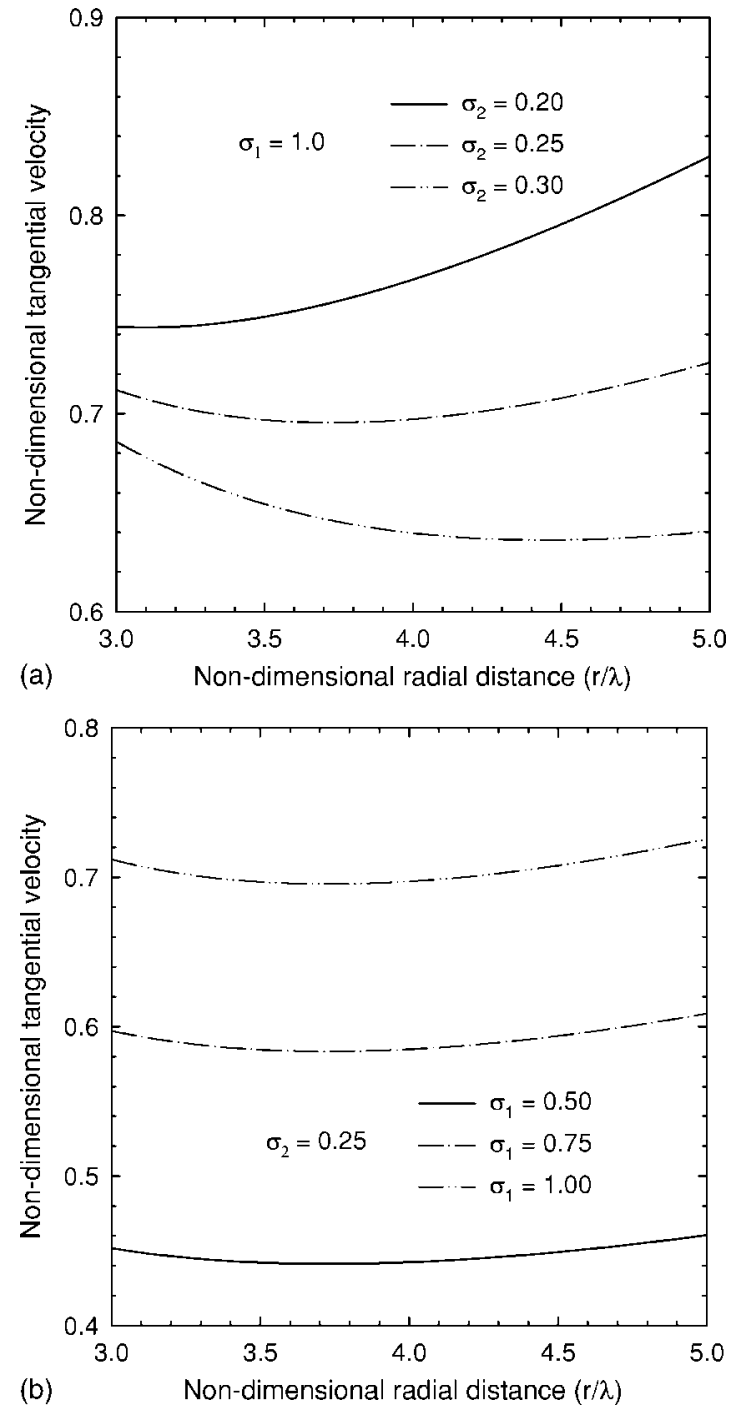

FIG. 4. Nondimensional velocity profiles when the accommodation coefficient at the stationary outer cylinder lies between 9/47 and 1/3.

$$
\frac{d^{2} u_{\theta}}{d r^{2}}=\frac{2}{(B-A) R_{1} r^{3}} .
$$

From the previous discussion, we know that $B-A>0$ and therefore Eq. (23) is always greater than zero, which implies that the velocity at $r=r^{*}$ is a minimum. Considering the previous two situations where the velocity is simply increasing or decreasing, it is evident that a partially inverted velocity profile will occur when the accommodation coefficient at the outer cylinder lies within the range

$$
2\left(1+\frac{\left(R_{1}^{2}+R_{2}^{2}\right) R_{2}}{2 \lambda R_{1}^{2}}\right)^{-1}<\sigma_{2}<2\left(1+\frac{R_{2}}{\lambda}\right)^{-1} .
$$

Figure 4 illustrates partially inverted velocity profiles for different combinations of accommodation coefficient. Figure 4(a) shows the case for $\sigma_{1}$ fixed at 1.0 and $\sigma_{2}$ varying between the previously defined limits of $9 / 47$ and $1 / 3$. It can be seen that the velocity minimum moves towards the outer cylinder as $\sigma_{2}$ increases. In contrast, Fig. 4(b) shows that the occurrence of a partially inverted velocity profile does not depend on the accommodation coefficient at the inner wall. 
For the specific case when $\sigma_{2}=0.25$, the location of the velocity minimum can be obtained from Eq. (21) and is given by $r^{*} / \lambda=5 \sqrt{5} / 3$. If the value of $\sigma_{2}$ is fixed, the location of the velocity minimum is independent of $\sigma_{1}$.

Maxwell ${ }^{16}$ predicted that when the accommodation coefficients at both walls approached zero (total specular reflection), the gas would rotate as a solid body, which implies $u_{\theta} \propto r$. To demonstrate this phenomenon, Eq. (9) can be rewritten in the following format:

$$
\begin{aligned}
u_{\theta}= & \omega_{1} r\left(1-\frac{\left(2-\sigma_{2}\right)}{\sigma_{2}} \frac{2 \lambda}{R_{2}}-\frac{R_{2}^{2}}{r^{2}}\right)\left[\left(1-\frac{\left(2-\sigma_{2}\right)}{\sigma_{2}} \frac{2 \lambda}{R_{2}}\right)\right. \\
& \left.-\frac{R_{2}^{2}}{R_{1}^{2}}\left(1+\frac{\left(2-\sigma_{1}\right)}{\sigma_{1}} \frac{2 \lambda}{R_{1}}\right)\right]^{-1} .
\end{aligned}
$$

When $\sigma_{1}$ and $\sigma_{2}$ approach zero at the same rate, i.e., $\sigma_{1}$ $=\sigma_{2} \rightarrow 0, u_{\theta}$ has the limit

$$
\lim _{\sigma_{1}=\sigma_{2} \rightarrow 0} u_{\theta}=\omega_{1} r\left(1+\frac{R_{2}^{3}}{R_{1}^{3}}\right)^{-1} .
$$

The current model is therefore in full agreement with Maxwell's original prediction. However, Aoki et al. ${ }^{3}$ have further shown that when the accommodation coefficients approach zero such that the ratio $\chi_{A}=\sigma_{2} / \sigma_{1}$ remains constant, the limiting value for the velocity $u_{\theta}$ will depend upon the ratio $\chi_{A}$ as follows:

$$
\lim _{\sigma_{2}=\chi_{A} \sigma_{1} \rightarrow 0} u_{\theta}=\omega_{1} r\left(1+\chi_{A} \frac{R_{2}^{3}}{R_{1}^{3}}\right)^{-1} .
$$

\section{B. Investigation of case II}

In case II, the inner cylinder is at rest while the outer cylinder rotates at angular speed $\omega_{2}$. The velocity gradient can be derived from Eq. (10) as

$$
\frac{d u_{\theta}}{d r}=\frac{1}{(B-A) R_{2}}\left(B+\frac{1}{r^{2}}\right) \text {. }
$$

From Eq. (8) we know that $B>0$ and from Eq. (15), $(B$ $-A)>0$, and therefore Eq. (28) will always be positive. This implies the velocity is an increasing function of radius and this feature is independent of both inner and outer accommodation coefficients. Hence, for case II, no inverted velocity profile will be observed. Figure 5(a) shows the case when $\sigma_{2}$ is fixed at 1.0 while $\sigma_{1}$ is varied whereas Fig. 5(b) shows the opposite case when $\sigma_{1}$ is fixed at 1.0 and $\sigma_{2}$ is varied. It can be observed that $\sigma_{2}$ has a greater impact on the velocity profile than $\sigma_{1}$.

\section{CONCLUSIONS}

The paper has presented an analytical evaluation of rarefied Couette flow between two concentric rotating cylinders. The Navier-Stokes equations, with boundary conditions derived from Maxwell's slip-flow model, were adapted to take into account the impact of a curved surface. The results are in close agreement with previous analytical solutions and in good qualitative agreement with available DSMC simulations. As the DSMC data show little variation

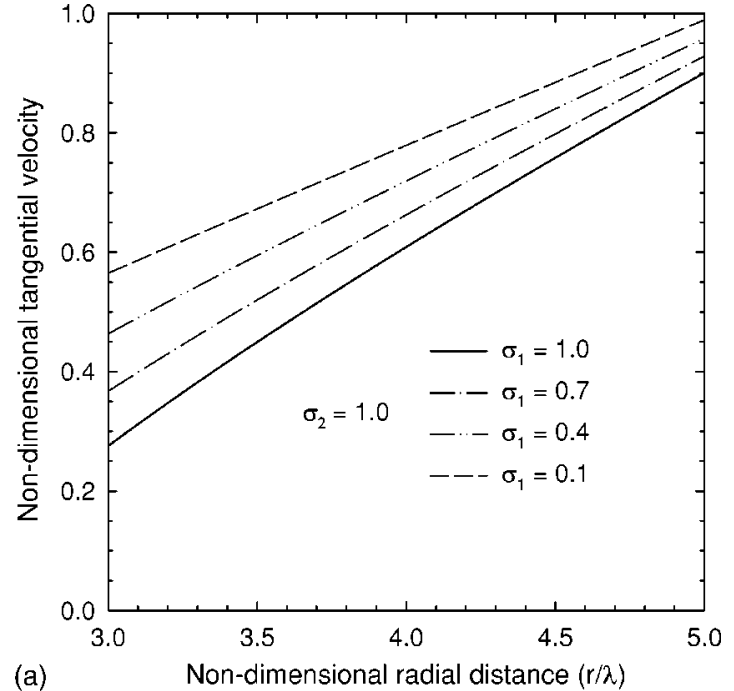

(a)

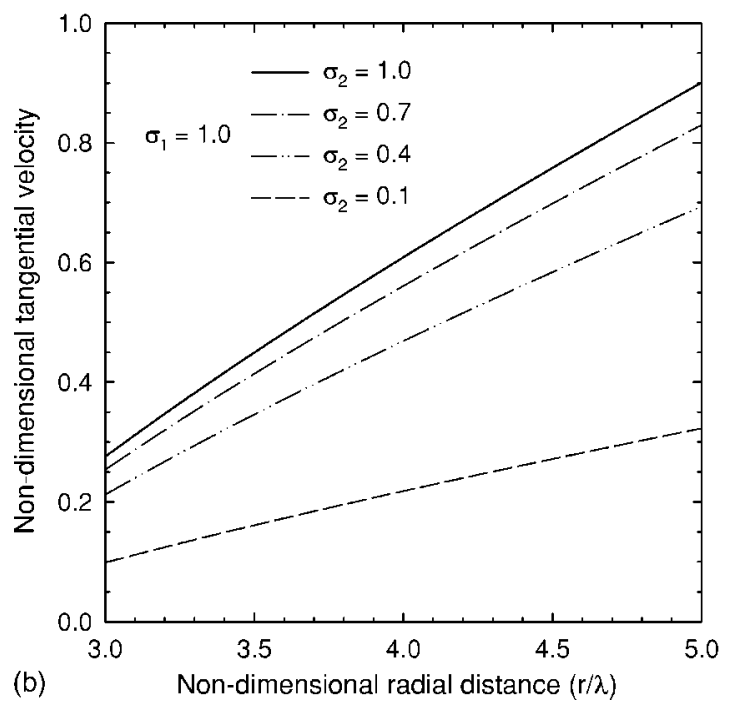

FIG. 5. Nondimensional velocity profiles for case II: inner cylinder at rest, outer cylinder rotating.

in density and temperature, the discrepancy between the analytical model and the DSMC solution can be attributed to the relatively high Knudsen number and the neglect of any Knudsen layer effects in the Navier-Stokes formulation. It has been shown that when the inner cylinder rotates and the outer cylinder is at rest, the phenomenon of velocity inversion depends solely on the accommodation coefficient of the stationary outer cylinder. The present analysis has identified the criteria that determine whether the velocity profile behaves normally or undergoes full or partial inversion. When the accommodation coefficient of the inner and outer cylinders have the same value, all velocity profiles pass through a common point whose location is independent of the value of the accommodation coefficient. For the case where the inner cylinder is at rest and the outer cylinder rotates, it has been shown that an inverted velocity profile will never occur.

\section{ACKNOWLEDGMENTS}

This work was carried out as part of the $\mu$ FAST program with support from the Medical Research Council under 
Grant No. 57719. Additional support was provided by the EPSRC under the auspices of Collaborative Computational Project 12 (CCP12).

${ }^{1}$ D. Einzel, P. Panzer, and M. Liu, "Boundary condition for fluid flow: Curved or rough surfaces," Phys. Rev. Lett. 64, 2269 (1990).

${ }^{2}$ K. W. Tibbs, F. Baras, and A. L. Garcia, "Anomalous flow profile due to the curvature effect on slip length," Phys. Rev. E 56, 2282 (1997).

${ }^{3}$ K. Aoki, H. Yoshida, T. Nakanishi, and A. L. Garcia, "Inverted velocity profile in the cylindrical Couette flow of a rarefied gas," Phys. Rev. E 68, 016302 (2003).

${ }^{4}$ J. C. Maxwell, "On stresses in rarefied gases arising from inequalities of temperature," Philos. Trans. R. Soc. London 170, 231 (1879).

${ }^{5}$ L. S. Pan, G. R. Liu, and K. Y. Lam, "Flow and load characteristics in a coaxial microbearing of finite length," J. Micromech. Microeng. 9, 270 (1999).

${ }^{6}$ L. Ren, K.-Q. Zhu, and X.-L. Wang, "Effects of the slip velocity boundary condition on the characteristics of microbearings," J. Micromech. Microeng. 14, 116 (2004).

${ }^{7}$ H. Schlichting, Boundary-Layer Theory, 7th ed. (McGraw-Hill, New York, 1979).

${ }^{8}$ D. A. Lockerby, J. M. Reese, D. R. Emerson, and R. W. Barber, "Velocity boundary condition at solid walls in rarefied gas calculations," Phys. Rev. E 70, 017303 (2004).

${ }^{9}$ R. W. Barber, Y. Sun, X. J. Gu, and D. R. Emerson, "Isothermal slip flow over curved surfaces," Vacuum 76, 73 (2004).

${ }^{10}$ S. K. Loyalka, N. Petrellis, and T. S. Storvick, "Some numerical results for the BGK model: Thermal creep and viscous slip problems with arbitrary accommodation at the surface," Phys. Fluids 18, 1094 (1975).

${ }^{11}$ M. Wakabayashi, T. Ohwada, and F. Golse, "Numerical analysis of the shear and thermal creep flows of a rarefied gas over the plane wall of a Maxwell-type boundary on the basis of the linearized Boltzmann equation for hard-sphere molecules," Eur. J. Mech. B/Fluids 15, 175 (1996).

${ }^{12}$ C. E. Siewert and F. Sharipov, "Model equations in rarefied gas dynamics: Viscous-slip and thermal-slip coefficients," Phys. Fluids 14, 4123 (2002).

${ }^{13}$ J. Maurer, P. Tabeling, P. Joseph, and H. Willaime, "Second-order slip laws in microchannels for helium and nitrogen," Phys. Fluids 15, 2613 (2003).

${ }^{14} \mathrm{C}$. Aubert and S. Colin, "High-order boundary conditions for gaseous flows in rectangular microducts," Microscale Thermophys. Eng. 5, 41 (2001).

${ }^{15}$ Y.-K. Hwang and J.-S. Heo, "Three-dimensional rarefied flows in rotating helical channels," J. Vac. Sci. Technol. A 19, 662 (2001).

${ }^{16}$ J. C. Maxwell, "The kinetic theory of gases," Nature (London), 26 July 1877 , p. 242. 